\title{
Infinite divisibility of Smith matrices
}

\author{
by
}

Shaofang Hong (Chengdu)

1. Introduction. Given an arithmetical function $f$, we denote by $f(a, b)$ and $f[a, b]$ the function $f$ evaluated at the greatest common divisor $(a, b)$ of positive integers $a$ and $b$ and at their least common multiple $[a, b]$ respectively. In 1875, Smith [18] showed his renowned result stating that the determinant of the $n \times n$ matrix $[f(i, j)]$, which has $f(i, j)$ as its $(i, j)$-entry, is the product $\prod_{k=1}^{n}(f * \mu)(k)$, where $\mu$ is the Möbius function and $f * \mu$ is the Dirichlet convolution of $f$ and $\mu$ defined for any integer $a \geq 1$ by $(f * \mu)(a)=\sum_{d \mid a} f(d) \mu(a / d)$, where $d$ runs over all positive divisors of $a$. Since then many generalizations and related results have been published. See, for instance, $[1,5-17,19]$. Later on, all such kind of matrices are called Smith matrices.

A positive semi-definite matrix $A=\left(a_{i j}\right)$ with $a_{i j} \geq 0$ for all $i$ and $j$ is called infinitely divisible if the fractional Hadamard power $A^{\circ r}=\left(a_{i j}^{r}\right)$ is positive semi-definite for every nonnegative real number $r$. Infinitely divisible matrices arise in several different contexts. Bhatia [3] and Bhatia and Kosaki [4] discussed this topic and presented some examples. Throughout this paper we always let $S=\left\{x_{1}, \ldots, x_{n}\right\}$ be any given set of $n$ distinct positive integers. Bourque and Ligh [5] showed that the power GCD matrix $\left(\left(x_{i}, x_{j}\right)^{r}\right)$ defined on $S$ is positive definite if $r>0$. From this one can see immediately that the power GCD matrix $\left(\left(x_{i}, x_{j}\right)^{r}\right)$ is infinitely divisible if $r>0$. It follows from [13] that the reciprocal power LCM matrix $\left(1 /\left[x_{i}, x_{j}\right]^{r}\right)$ is positive definite, and hence is infinitely divisible if $r>0$. Note that an LCM matrix $\left(\left[x_{i}, x_{j}\right]\right)$ and a reciprocal GCD matrix $\left(1 /\left[x_{i}, x_{j}\right]\right)$ may be singular (see $[9,11])$. Bourque and Ligh $[5]$ proved that the matrix $\left(f\left(x_{i}, x_{j}\right)\right)$

2000 Mathematics Subject Classification: Primary 11C20, 11A25.

Key words and phrases: multiplicative function, $l$ th Dirichlet convolution, Smith matrix, infinite divisibility.

The research was supported by Program for New Century Excellent Talents in University Grant \# NCET-06-0785. 
is positive definite if $f$ is in

$$
\tilde{\mathcal{C}}_{S}:=\{f \mid(f * \mu)(d)>0 \text { whenever } d \mid x \text { for any } x \in S\} .
$$

In this paper, we consider a natural class of arithmetical functions

$$
\mathcal{C}_{S}:=\{f \mid(f * \mu)(d) \geq 0 \text { whenever } d \mid x \text { for any } x \in S\} .
$$

Using a continuity argument we show that the matrix $\left(f\left(x_{i}, x_{j}\right)\right)$ is positive semi-definite if $f \in \mathcal{C}_{S}$. Consequently, such a matrix is infinitely divisible if $f \in \mathcal{C}_{S}$ is multiplicative. But it may not be infinitely divisible if $f$ is not multiplicative. We also show that the matrices $\left(1 / f\left[x_{i}, x_{j}\right]\right)$ and $\left(f\left(x_{i}, x_{j}\right) / f\left[x_{i}, x_{j}\right]\right)$ are infinitely divisible if $f \in \mathcal{C}_{S}$ is multiplicative, where we make the convention $1 / f(a):=0$ if $f(a)=0$. Finally, we extend these results to the Dirichlet convolution case which produces infinitely many examples of infinitely divisible matrices. Our results extend those of Bourque, Ligh, Bhatia, Hong, Lee, Lindqvist and Seip. We refer the readers to [2] for the basic elementary concepts and facts from number theory.

2. Lemmas and theorems. Using [8] and a continuity argument we show the following result.

Lemma 2.1. If $f \in \mathcal{C}_{S}$, then the $n \times n$ matrix $\left(f\left(x_{i}, x_{j}\right)\right)$ is positive semi-definite.

Proof. Let $f \in \mathcal{C}_{S}$ and pick $\varepsilon>0$ and $\bar{f} \in \tilde{\mathcal{C}}_{S}$. Then it is easy to see that $f+\varepsilon \bar{f} \in \tilde{\mathcal{C}}_{S}$. For an arithmetical function $g$ and $1 \leq k \leq n$, let

$$
\alpha_{g}\left(x_{k}\right):=\sum_{\substack{d \mid x_{k} \\ d \nmid x_{t}, x_{t}<x_{k}}}(g * \mu)(d) .
$$

By Theorem 1 of [8],

$$
\operatorname{det}\left((f+\varepsilon \bar{f})\left(x_{i}, x_{j}\right)\right) \geq \prod_{k=1}^{n} \alpha_{f+\varepsilon \bar{f}}\left(x_{k}\right) .
$$

Note that both sides of (1) are polynomials in $\varepsilon$. Moreover, the constant terms of the left and right hand sides are, respectively, $\operatorname{det}\left(f\left(x_{i}, x_{j}\right)\right)$ and $\prod_{k=1}^{n} \alpha_{f}\left(x_{k}\right)$. Since (1) holds for any $\varepsilon>0$, letting $\varepsilon \rightarrow 0$ we obtain

$$
\operatorname{det}\left(f\left(x_{i}, x_{j}\right)\right) \geq \prod_{k=1}^{n} \alpha_{f}\left(x_{k}\right) .
$$

For any $1 \leq l \leq n$, since $f \in \mathcal{C}_{S}$, the inequality (2) implies that the determinant of any principal submatrix of order $l$ of $\left(f\left(x_{i}, x_{j}\right)\right)$ is nonnegative. This shows that the matrix $\left(f\left(x_{i}, x_{j}\right)\right)$ is positive semi-definite. 
Associated to any nonnegative real number $r$ and the arithmetical function $f$ such that $f(m) \geq 0$ for any positive integer $m$, we define the arithmetical function $f^{r}$ by $f^{r}(m):=f(m)^{r}$ for any positive integer $m$.

Lemma 2.2. Let $f$ be a multiplicative function such that $f \in \mathcal{C}_{S}$. Then $f^{r} \in \mathcal{C}_{S}$ for any nonnegative real number $r$.

Proof. Since $f$ is multiplicative, so is $f^{r}$. Hence $f^{r} * \mu$ is multiplicative. Therefore it is sufficient to show that $\left(f^{r} * \mu\right)\left(p^{e}\right) \geq 0$ for any prime $p$ and any positive integer $e$ such that $p^{e}$ divides some $x \in S$.

Evidently,

$$
(f * \mu)\left(p^{e}\right)\left(f^{r} * \mu\right)\left(p^{e}\right)=\left(f\left(p^{e}\right)-f\left(p^{e-1}\right)\right)\left(f\left(p^{e}\right)^{r}-f\left(p^{e-1}\right)^{r}\right) .
$$

Notice that $f\left(p^{e}\right)=\sum_{t=0}^{e}(f * \mu)\left(p^{t}\right) \geq 0$ for any nonnegative integer $e$ since $f \in \mathcal{C}_{S}$ implies that $(f * \mu)\left(p^{t}\right) \geq 0$ for any integer $0 \leq t \leq e$. Since $r \geq 0$, we have $f\left(p^{e}\right)^{r} \geq f\left(p^{e-1}\right)^{r} \geq 0\left(\right.$ resp. $\left.0 \leq f\left(p^{e}\right)^{r} \leq f\left(p^{e-1}\right)^{r}\right)$ if $f\left(p^{e}\right) \geq f\left(p^{e-1}\right)$ (resp. $\left.f\left(p^{e}\right) \leq f\left(p^{e-1}\right)\right)$. Thus

$$
\left(f\left(p^{e}\right)-f\left(p^{e-1}\right)\right)\left(f\left(p^{e}\right)^{r}-f\left(p^{e-1}\right)^{r}\right) \geq 0 .
$$

But $(f * \mu)\left(p^{e}\right) \geq 0$ since $f \in \mathcal{C}_{S}$. Then by $(3)$ and $(4),\left(f^{r} * \mu\right)\left(p^{e}\right) \geq 0$. So $f^{r} \in \mathcal{C}_{S}$ as required.

THEOREM 2.1. If $f$ is multiplicative and $f \in \mathcal{C}_{S}$, then the $n \times n$ matrices $\left(f\left(x_{i}, x_{j}\right)\right),\left(1 / f\left[x_{i}, x_{j}\right]\right)$ and $\left(f\left(x_{i}, x_{j}\right) / f\left[x_{i}, x_{j}\right]\right)$ are infinitely divisible.

Proof. Since $f \in \mathcal{C}_{S}$ is multiplicative, by Lemma 2.2 we know that $f^{r} \in \mathcal{C}_{S}$ for any $r \geq 0$. It then follows from Lemma 2.1 that for any $r \geq 0$, the $n \times n$ matrix $\left(f^{r}\left(x_{i}, x_{j}\right)\right)$ is positive semi-definite. So the matrix $\left(f\left(x_{i}, x_{j}\right)\right)$ is infinitely divisible. One can easily check the following identities:

$$
\left(\frac{1}{f^{r}\left[x_{i}, x_{j}\right]}\right)=D\left(f^{r}\left(x_{i}, x_{j}\right)\right) D, \quad\left(\frac{f^{r}\left(x_{i}, x_{j}\right)}{f^{r}\left[x_{i}, x_{j}\right]}\right)=D\left(f^{2 r}\left(x_{i}, x_{j}\right)\right) D,
$$

where $D=\operatorname{diag}\left(1 / f^{r}\left(x_{1}\right), \ldots, 1 / f^{r}\left(x_{n}\right)\right)$. Thus the matrices $\left(1 / f^{r}\left[x_{i}, x_{j}\right]\right)$ and $\left(f^{r}\left(x_{i}, x_{j}\right) / f^{r}\left[x_{i}, x_{j}\right]\right)$ are positive semi-definite if $r \geq 0$. In other words, $\left(1 / f\left[x_{i}, x_{j}\right]\right)$ and $\left(f\left(x_{i}, x_{j}\right) / f\left[x_{i}, x_{j}\right]\right)$ are infinitely divisible.

REMARK. It should be pointed out that the condition that $f$ is multiplicative is necessary. Otherwise the conclusion may be false. For example, let $S=\{6,10,15\}$ and let $f$ be defined by $f(1)=f(3)=0, f(10)=3$ and $f(m)=1$ for $m \neq 1,3,10$. It is clear that $f \in \mathcal{C}_{S}$ and $f$ is not multiplicative. Obviously,

$$
\left(f\left(x_{i}, x_{j}\right)\right)=\left(\begin{array}{ccc}
1 & 1 & 0 \\
1 & 3 & 1 \\
0 & 1 & 1
\end{array}\right)
$$

is positive semi-definite. One can easily prove that $\left(f\left(x_{i}, x_{j}\right)\right)^{\circ r}$ is positive 
semi-definite if and only if $r \geq \log 2 / \log 3$. Hence the matrix $\left(f\left(x_{i}, x_{j}\right)\right)$ is not infinitely divisible.

Let $l \geq 0$ be an integer. For any arithmetical function $f$, define its $l$ th Dirichlet convolution, denoted by $f^{(l)}$, inductively as follows: $f^{(0)}:=\delta$ and $f^{(l)}:=f^{(l-1)} * f$ if $l \geq 1$, where $\delta$ is the function defined for any positive integer $m$ by $\delta(m):=1$ if $m=1$, and 0 otherwise. Evidently, $f * \delta=f$ for any arithmetical function $f$ and $f^{(l)}:=\underbrace{f * \cdots * f}_{l \text { times }}$.

Let $\mathbb{Z}_{>0}$ denote the set of positive integers.

Lemma 2.3. Let $c \geq 1$ and $d \geq 0$ be integers. If $f_{1}, \ldots, f_{c} \in \mathcal{C}_{S}$ are distinct arithmetical functions and $\left(l_{1}, \ldots, l_{c}\right) \in \mathbb{Z}_{>0}^{c}$ satisfies $l_{1}+\cdots+l_{c}>d$, then $f_{1}^{\left(l_{1}\right)} * \cdots * f_{c}^{\left(l_{c}\right)} * \mu^{(d)} \in \mathcal{C}_{S}$.

Proof. Clearly it is sufficient to prove that for any integer $l>d$ and any (not necessarily distinct) arithmetical functions $g_{1}, \ldots, g_{l} \in \mathcal{C}_{S}$, we have $g_{1} * \cdots * g_{l} * \mu^{(d)} \in \mathcal{C}_{S}$. Indeed, for any $x \in S$ and any $m \mid x$, since $l \geq d+1$, we have

$$
\begin{aligned}
& \left(\left(g_{1} * \cdots * g_{l} * \mu^{(d)}\right) * \mu\right)(m) \\
= & \left(g_{1} * \cdots * g_{l} * \mu^{(d+1)}\right)(m) \\
= & \left(\left(g_{1} * \mu\right) * \cdots *\left(g_{d} * \mu\right) *\left(g_{d+1} * \mu\right) * g_{d+2} * \cdots * g_{l}\right)(m) \\
= & \sum_{\substack{m_{1} \cdots m_{l}=m \\
\left(m_{1}, \ldots, m_{l}\right) \in \mathbb{Z}_{>0}^{l}}}\left(g_{1} * \mu\right)\left(m_{1}\right) \cdots\left(g_{d+1} * \mu\right)\left(m_{d+1}\right) g_{d+2}\left(m_{d+2}\right) \cdots g_{l}\left(m_{l}\right) .
\end{aligned}
$$

For $1 \leq i \leq d+1$, since $g_{i} \in \mathcal{C}_{S}$ and $m_{i} \mid x$, we have $\left(g_{i} * \mu\right)\left(m_{i}\right) \geq 0$. On the other hand, for $d+2 \leq j \leq l, g_{j} \in \mathcal{C}_{S}$ together with $m_{j} \mid x$ implies that $g_{j}\left(m_{j}\right)=\sum_{d^{\prime} \mid m_{j}}\left(g_{j} * \mu\right)\left(d^{\prime}\right) \geq 0$. It then follows from (5) that $\left(\left(g_{1} * \cdots * g_{l} * \mu^{(d)}\right) * \mu\right)(m) \geq 0$ as desired.

From Theorem 2.1 and Lemma 2.3 we deduce immediately the following more general result.

Theorem 2.2. Let $c \geq 1$ and $d \geq 0$ be integers. If $f_{1}, \ldots, f_{c} \in \mathcal{C}_{S}$ are distinct and multiplicative and $\left(l_{1}, \ldots, l_{c}\right) \in \mathbb{Z}_{>0}^{c}$ satisfies $l_{1}+\cdots+l_{c}>d$, then the three $n \times n$ matrices

$$
\begin{gathered}
\left(\left(f_{1}^{\left(l_{1}\right)} * \cdots * f_{c}^{\left(l_{c}\right)} * \mu^{(d)}\right)\left(x_{i}, x_{j}\right)\right), \quad\left(\frac{1}{\left(f_{1}^{\left(l_{1}\right)} * \cdots * f_{c}^{\left(l_{c}\right)} * \mu^{(d)}\right)\left[x_{i}, x_{j}\right]}\right), \\
\left(\frac{\left(f_{1}^{\left(l_{1}\right)} * \cdots * f_{c}^{\left(l_{c}\right)} * \mu^{(d)}\right)\left(x_{i}, x_{j}\right)}{\left(f_{1}^{\left(l_{1}\right)} * \cdots * f_{c}^{\left(l_{c}\right)} * \mu^{(d)}\right)\left[x_{i}, x_{j}\right]}\right)
\end{gathered}
$$

are infinitely divisible. 
In particular, we have

THEOREM 2.3. If $f \in \mathcal{C}_{S}$ is multiplicative and $l>d \geq 0$ are integers, then the three $n \times n$ matrices

$$
\left(\left(f^{(l)} * \mu^{(d)}\right)\left(x_{i}, x_{j}\right)\right), \quad\left(\frac{1}{\left(f^{(l)} * \mu^{(d)}\right)\left[x_{i}, x_{j}\right]}\right), \quad\left(\frac{\left(f^{(l)} * \mu^{(d)}\right)\left(x_{i}, x_{j}\right)}{\left(f^{(l)} * \mu^{(d)}\right)\left[x_{i}, x_{j}\right]}\right)
$$

are infinitely divisible.

3. Examples. In this section, we give some examples to illustrate our main results.

ExAmple 3.1. Let $\xi_{\varepsilon}$ be defined by $\xi_{\varepsilon}(m)=m^{\varepsilon}$ for any integer $m \geq 1$. It is easy to check that $\xi_{\varepsilon} \in \mathcal{C}_{S}$ for any set $S$ of positive integers and any $\varepsilon \geq 0$. By Theorem 2.1, the matrices

$$
\left(\left(x_{i}, x_{j}\right)^{\varepsilon}\right), \quad\left(\frac{1}{\left[x_{i}, x_{j}\right]^{\varepsilon}}\right), \quad\left(\frac{\left(x_{i}, x_{j}\right)^{\varepsilon}}{\left[x_{i}, x_{j}\right]^{\varepsilon}}\right)
$$

are infinitely divisible for any nonnegative real number $\varepsilon$. Note that HongLoewy [14], Hong-Lee [13] and Lindqvist and Seip [16] investigated the asymptotic behavior of the eigenvalues of the above three matrices respectively.

EXAMPLE 3.2. Let $J_{\varepsilon}:=\xi_{\varepsilon} * \mu$ be the generalized Jordan function. Since $\left(J_{\varepsilon} * \mu\right)(p)=p^{\varepsilon}-2 \geq 2^{\varepsilon}-2 \geq 0$ for any prime $p$ and any real number $\varepsilon \geq 1$, we have $J_{\varepsilon} \in \mathcal{C}_{S}$ for any set $S$ of positive integers and any $\varepsilon \geq 1$. By Theorem 2.1, the matrices

$$
\left(J_{\varepsilon}\left(x_{i}, x_{j}\right)\right), \quad\left(\frac{1}{J_{\varepsilon}\left[x_{i}, x_{j}\right]}\right), \quad\left(\frac{J_{\varepsilon}\left(x_{i}, x_{j}\right)}{J_{\varepsilon}\left[x_{i}, x_{j}\right]}\right)
$$

are infinitely divisible for any real number $\varepsilon \geq 1$.

Example 3.3. Let $\xi_{\varepsilon}$ and $J_{\varepsilon}:=\xi_{\varepsilon} * \mu$ be defined as above. Then by Theorem 2.2, the matrices

$$
\begin{gathered}
\left(\left(\xi_{\varepsilon}^{(l)} * J_{\delta}^{(t)} * \mu^{(d)}\right)\left(x_{i}, x_{j}\right)\right), \quad\left(\frac{1}{\left(\xi_{\varepsilon}^{(l)} * J_{\delta}^{(t)} * \mu^{(d)}\right)\left[x_{i}, x_{j}\right]}\right), \\
\left(\frac{\left(\xi_{\varepsilon}^{(l)} * J_{\delta}^{(t)} * \mu^{(d)}\right)\left(x_{i}, x_{j}\right)}{\left(\xi_{\varepsilon}^{(l)} * J_{\delta}^{(t)} * \mu^{(d)}\right)\left[x_{i}, x_{j}\right]}\right)
\end{gathered}
$$

are infinitely divisible for any real numbers $\varepsilon \geq 0$ and $\delta \geq 1$ and any nonnegative integers $l, t$ and $d$ such that $l+t>d$.

Finally, we remark that Hong [12] and Hong and Loewy [15] studied the asymptotic behavior of the eigenvalues of matrices associated with arithmetical functions including all the matrices in Examples 3.1-3.3 as special cases. 
Acknowledgements. The author would like to thank the anonymous referee for valuable comments and suggestions.

\section{References}

[1] T. M. Apostol, Arithmetical properties of generalized Ramanujan sums, Pacific J. Math. 41 (1972), 281-293.

[2] - Introduction to Analytic Number Theory, Springer, New York, 1976.

[3] R. Bhatia, Infinitely divisible matrices, Amer. Math. Monthly 113 (2006), 221-235.

[4] R. Bhatia and H. Kosaki, Mean matrices and infinite divisibility, Linear Algebra Appl. 424 (2007), 36-54.

[5] K. Bourque and S. Ligh, Matrices associated with classes of arithmetical functions, J. Number Theory 45 (1993), 367-376.

[6] P. Codecá and M. Nair, Calculating a determinant associated with multiplicative functions, Boll. Un. Mat. Ital. B (8) 5 (2002), 545-555.

[7] T. Hilberdink, Determinants of multiplicative Toeplitz matrices, Acta Arith. 125 (2006), 265-284.

[8] S. F. Hong, Bounds for determinants of matrices associated with classes of arithmetical functions, Linear Algebra Appl. 281 (1998), 311-322.

[9] - On the Bourque-Ligh conjecture of least common multiple matrices, J. Algebra 218 (1999), 216-228.

[10] -, Gcd-closed sets and determinants of matrices associated with arithmetical functions, Acta Arith. 101 (2002), 321-332.

[11] —, Nonsingularity of least common multiple matrices on gcd-closed sets, J. Number Theory 113 (2005), 1-9.

[12] - , Asymptotic behavior of the largest eigenvalue of matrices associated with completely even functions (mod $r$ ), Asian-Eur. J. Math. 1 (2008), 225-235.

[13] S. F. Hong and E. K. S. Lee, Asymptotic behavior of eigenvalues of reciprocal power LCM matrices, Glasgow Math. J. 50 (2008), 163-174.

[14] S. F. Hong and R. Loewy, Asymptotic behavior of eigenvalues of greatest common divisor matrices, ibid. 46 (2004), 551-569.

[15] - - - Asymptotic behavior of the smallest eigenvalue of matrices associated with completely even functions (mod $r$ ), preprint, arXiv:0808.4118v1.

[16] P. Lindqvist and K. Seip, Note on some greatest common divisor matrices, Acta Arith. 84 (1998), 149-154.

[17] P. J. McCarthy, A generalization of Smith's determinant, Canad. Math. Bull. 29 (1988), 109-113.

[18] H. J. S. Smith, On the value of a certain arithmetical determinant, Proc. London Math. Soc. 7 (1875-1876), 208-212.

[19] A. Wintner, Diophantine approximations and Hilbert's space, Amer. J. Math. 66 (1944), 564-578.

Mathematical College

Sichuan University

Chengdu 610064, P.R. China

E-mail: sfhong@scu.edu.cn, s-f.hong@tom.com, hongsf02@yahoo.com 